\title{
Upper Bound on Blocking Probability for Vertically Stacked Optical Banyan Networks with Link Failures and Given Crosstalk Constraint
}

\author{
M. R. Khandker ${ }^{\dagger}$ and B. Sultana \\ Department of Applied Physics and Electronic Engineering, University of Rajshahi, Bangladesh
}

Received 24 September 2008, accepted in final revised form 16 July 2009

\begin{abstract}
Vertical stacking of multiple copies of an optical banyan network is a novel scheme for building nonblocking optical switching networks. The resulting network, namely vertically stacked optical banyan (VSOB) network, preserves all the properties of the banyan network, but increases the hardware cost significantly under first order crosstalk-free constraint. However, stringent crosstalk constraint may not always be necessary. Considering the fact that some designer may want to compromise the blocking probability and crosstalk constraint to a certain degree with the hardware cost, blocking behaviour of such VSOB networks have been analyzed to studying network performance and finding a graceful compromise between hardware costs and blocking probability under or without crosstalk constraint. In this paper, we present the simulation results for upper bound on blocking probability of VSOB networks with link failures and given degree of crosstalk constraint. We show how crosstalk adds a new dimension to the performance analysis on a VSOB networks. The simulation results presented in this paper can guide network designer in finding the trade-off among the blocking probability, the degree of crosstalk and the hardware cost in terms of vertical copies of banyan network in the presence of link failures.
\end{abstract}

Keywords: Banyan networks; Blocking probability; Vertical stacking; Link-failures; Crosstalk.

C) 2009 JSR Publications. ISSN: 2070-0237 (Print); 2070-0245 (Online). All rights reserved.

DOI: 10.3329/jsr.v1i3.1191 J. Sci. Res. 1 (3), 484-494 (2009)

\section{Introduction}

Optical mesh networks are considered more capacity-efficient and survivable for serving as the backbones for next generation internet. A key network element equipped with a switching node of optical mesh networks is the optical switch, which has the capability of switching huge data at an ultra-high speed. The basic $2 \times 2$ switching element (SE) in a large optical switching network is usually a directional coupler (DC) [1, 8]. DC's can switch multiple wavelengths at the same time, which is important for the future optical cross-connects (OXCs).

\footnotetext{
${ }^{\dagger}$ Corresponding author: khandker@ru.ac.bd
} 
Crosstalk is an intrinsic shortcoming of the DC. It is the effect of the undesirable coupling between the signals carried in the two waveguides of the coupler $[1,2]$. When two optical signals meet at a DC, a small portion of the signal power will be directed to the unintended output channel. Crosstalk suppression becomes particularly important in networks, where a signal propagates through many nodes and accumulates crosstalk from different elements at each node from the system view. Networks are very likely to contain amplifiers and to be limited by signal-spontaneous beat noise. For example, if we have 10 interfering equal-power crosstalk elements, each producing intra-channel crosstalk, then we must have a crosstalk suppression of below $35 \mathrm{~dB}$ in each element, in order to have an overall penalty of less than $1 \mathrm{~dB}$ [8]. Thus, Crosstalk reduction is an important issue in designing the systems that are based on DC's. The crosstalk issue can be tackled at either the device level or the system level. The two methods complement each other. The focus of this paper is on the system-level approach. As will be seen, crosstalk adds a new dimension to the theory of building a nonblocking and negligible blocking switching network.

Banyan networks [3-5, 9] are a class of attractive switching structures for constructing DC-based optical switches, because they have a smaller and exactly the same number of SEs along any path between an input-output pair such that an absolutely loss uniformity and smaller attenuation of optical signals are guaranteed in this class of switching networks. However, with the banyan topology only a unique path can be found from each network input to each network output, in which the network is degraded as a blocking one. The general scheme for building banyan-based nonblocking optical switching networks is to vertically stack the multiple copies of regular optical banyan network [7, 11] as illustrated in Fig. 1.

Conventionally, blocking happens when two connections intend to use the same link, which is referred to as link-blocking. In optical banyan networks, however, there is another type of connection-related blocking, which occurs when some paths (including the new one) violate the crosstalk-free constraint after adding the new connection. In such a situation, the connection is not allowed to be allocated even if the path is available. We refer to this second type of blocking as crosstalk-blocking throughout the paper. It is the combination of the two types of blocking that makes the design principle different. We use VSOB to denote the optical switching networks built on vertical stacking scheme of banyan network.

Numerous result are available for VSOB networks, such as [7], and their main focus has been on determining the maximum number of stacked copies (planes) required for a nonblocking VSOB networks without link-failures if conservative routing algorithm is used for routing a request to a plane. These results indicate that VSOB structure, although is attractive, usually requires either a high hardware cost or a relatively larger depth for building a nonblocking network.

Due to the increasing importance and requirement for fault-tolerance in optical switches for large mesh WDM networks, performance analysis on VSOB networks at the presence of probability of link failures becomes critical for the practical adoption of the 
VSOB networks in the current internet applications. Blocking behavior analysis of a network is an efficient approach to studying network performance and finding desirable trade-off between hardware costs and blocking probability. Some analytical models have been developed to understand the blocking behaviors of vertical stacked optical banyan networks that do not meet the nonblocking conditions under crosstalk-free constraint (i.e., with fewer stacked copies than required by nonblocking condition) [7,10,11]. However, to the best of our knowledge, no results are available for evaluating the probabilities of VSOB networks with link failures under various degree of crosstalk constraint. As the first important step towards the blocking behavior analysis of general VSOB networks under any crosstalk constraint, we presented the blocking probabilities of VSOB networks under crosstalk constraint with $c=1,2$ and 3, where $c$ is the degree of crosstalk, and their upper bound with respect to the number of planes in the networks. The simulation results can guide network designers to make a compromise between the hardware cost and the blocking probability of a VSOB network under different degree of crosstalk.

The rest of the paper is organized as follows: section 2 provides preliminaries that facilitate our further discussions. Section 3 presents our contribution, whereas section 4 concludes the paper.

\section{Preliminaries}

A typical $N \times N$ banyan network consists of $\log _{2} N$ stages, each containing $N / 22 \times 2$ SEs. It has a unique path between an input-output pair, and a basic technique for creating multiple paths between an input-output pair is the vertical stacking of multiple copies of the banyan network. The optical version of this type banyan networks are called VSOB networks as shown in Fig. 1 (b).

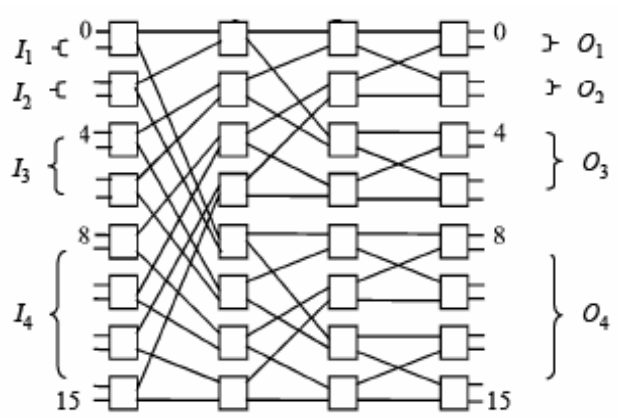

(a)

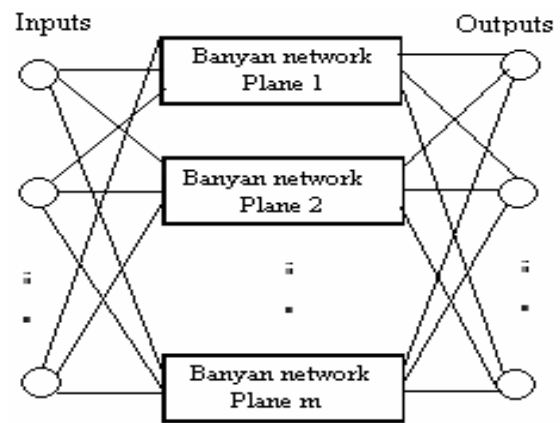

(b)

Fig. 1. (a) A 16×16 banyan network. (b) A vertically stacked optical banyan (VSOB) network.

Due to the topological symmetry, all paths in banyan networks have the same property in terms of blocking. We define the blocking probability to be the probability that a feasible connection request is blocked, where a feasible connection request is a connection request between an idle input port and an idle output port of the network. 
Without loss of generality, we focus on the path between the first input and the first output (which is termed as the tagged path hereafter). All the SEs and links on the tagged path are called tagged SEs and tagged links respectively. The stages of SEs are numbered from left (stage 1) to right (stage $\log _{2} N$ ) and the stages of links are also numbered from left (stage 1) to right (stage $\log _{2} N+1$ ). For the tagged path, an input intersecting set $I_{i}=\left\{2^{i-}\right.$ $\left.1,2^{i-1}+1, \ldots, 2^{i}-1\right\}$ at stage $i$ is defined as the set of all inputs that intersect a tagged SE at stage $i$. Likewise, an output intersecting set $O_{i}=\left\{2^{i-1}, 2^{i-1}+1, \ldots, 2-1\right\}$ associated with stage $i$ contains all the outputs that intersect a tagged SE at stage $\log _{2} N-i+1$.

When two light signals go through an SE simultaneously, crosstalk is generated in the signal at the SE. Such SE is referred to as a crosstalk SE (CSE). The degree of crosstalk of the switching system is defined as the number of CSE's allowed along a path.

A restricted SE (RSE) is a $2 \times 2$ SE which carries only one light signal at a time. Although crosstalk at an RSE is very small, it may not be entirely zero. For example, when a light signal passes through an RSE, a small portion of the signal will leave at the other unintended output channel. This stray signal can arrive at the input of the next stage $\mathrm{SE}$ and generate some crosstalk. Since crosstalk generated by the stray signal is much smaller than the regular crosstalk, we will ignore it in our analysis. The upper bounds for blocking probability in terms of number of planes for such nonblocking networks without any crosstalk have been presented in [7] as,

$$
T \geq\left\{\begin{array}{c}
2 \sqrt{N}-1 \text { when } \log _{2} N \text { is even } \\
(3 / 2) \sqrt{2 N}-1 \text { when } \log _{2} N \text { is odd }
\end{array},\right.
$$

where $T$ is the number planes. Eq. (1) states that a $16 \times 16$ first-order crosstalk-free VSOB network needs 7 or more planes to ensure strictly nonblocking condition.

Following the typical assumption as in $[7,10]$ on probabilistic analysis of multistage interconnection networks, we neglect the correlation among signals arriving at input and output ports, and consider that the statuses (busy or idle) of individual input and output ports in the network are independent. This assumption is justified by the fact that the correlation among signals at inputs and outputs, though exists for fixed communication patterns, become negligible for arbitrary communication patterns in large size networks, which is the trend of future optical switching networks that can switch huge data at high speed.

\subsection{VSOB network with link failures}

Eq. (1) assumes all the links in the VSOB networks are good. However, a link may be broken during the manufacturing time, and a connection request may also be blocked by link failures in a faulty VSOB network. We assume that the links in VSOB networks may fail independently and these failures are permanent. Thus, both crosstalk-blocking and failure-blocking should be fully considered in the blocking analysis of a faulty VSOB network as illustrated in Fig. 2 for a $8 \times 8$ network. 


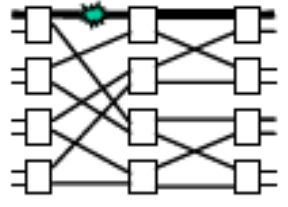

(a)

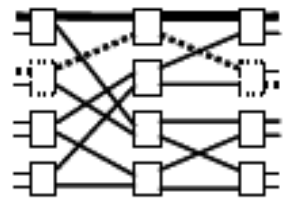

(b)

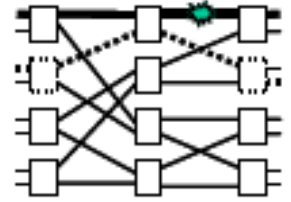

(c)

Fig. 2. Blocking in a VSOB network, (a) Failure-blocking, (b) Crosstalk-blocking, (c) Combination of failure-blocking and crosstalk-blocking.

\subsection{Upper bound on number on blocking probability}

\subsubsection{Simulation model}

The network simulator we developed consists of six major modules as shown in Fig. 3.

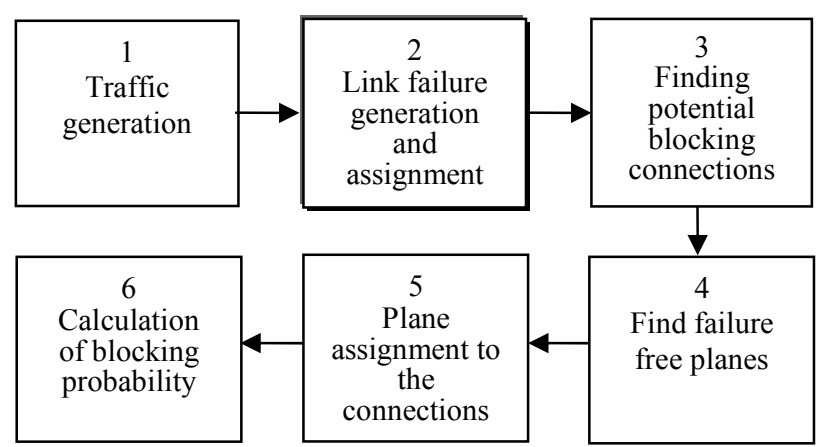

Fig. 3. Block diagram of network simulator.

We consider here the permutation request as the traffic. Due to the symmetric architecture of $\operatorname{VSOB}(N, T)$ network, every connection request has the same probability to be blocked. In our simulation, we fix the connection request of input-output pair $0-0$ and investigate the blocking probability of this connection request only that may result by other contentious connections. The traffic generation module randomly generates a permutation request for the $\operatorname{VSOB}(N, T)$ network based on the workload $r$ (here workload $r$ is defined as the occupancy probability of a port). The link failure generation and assignment module generates link failures based on the given $p f r$ (here $p f r$ is defined as the probability that a link is failed or broken) and then assign those failures randomly to different links.

In this section we present some definitions which will be necessary in the discussion of our simulation procedure: 
Definition 1. Set of blocking connections, $C_{b c}$ : The connections that are potential to blocking the tagged path.

Definition 2. Set of crosstalk-blocking connections, $C_{c b c}$ : The connections that are potential to resulting in crosstalk in the tagged path. $C_{c b c}$ is a subset of $C_{b c}$.

Definition 3. Set of failure free planes, $P_{i}$ : List of planes that are free from linkfailures on the path of each $C_{b c}$. Each entry has two fields. The first field represents the plane number and the second field contains a list of connections that can be established in this plane.

Definition 4. Amount of crosstalk, C: Number of crosstalk SEs (CSE) along the tagged path.

Definition 5. Amount of maximum allowed crosstalk, $C_{m}$ : Maximum number of CSE that is allowed along the tagged path.

Module 1 generates a permutation and initializes the switch network. Module 2 embeds failures into links randomly chosen; each link has pfr probability to be failed. In module 3 first we check all the planes and make a list of planes, say $P_{\text {tagged }}$, in which no links on the tagged path are failed or broken. Then we find $C_{b c}$ which is determined by the following relation:

$$
I_{i}+O_{j}<\log _{2} N+2
$$

Here, input $i$ has originated from input intersecting group $I_{i}$ and destined to output $j$ that belongs to output intersecting group $O_{j}$ (see Fig. (a)).

$C_{c b c}$ has been constructed from $C_{\mathrm{bc}}$ such that each connection ij must satisfy the following relation

$$
I_{i}+O_{j}=\log _{2} N+1
$$

Module 4 finds $P_{i}$ from $P_{\text {tagged }}$.

The plane assignment module assigns connection requests to different planes in the following way. First, the tagged path is assigned to a plane (say, $P_{t g}$ ) randomly chosen from $P_{\mathrm{i}}$. Then a request from $C_{c b c}$ is chosen and assigned to $P_{t g}$ provided that $C \leq C_{m}$. Then all the requests from $C_{c b c}$ are tried to establish in $P_{t g}$ one after another satisfying this crosstalk constraint. Let $K$ be the subset of $C_{c b c}$ that is successfully establish in $P_{t g}$. Now each connection from $C_{b c}-K$ is tried to assign to a separate plane from $P_{i}$ excluding the $P_{t g}$. This plane assignment algorithm ensures the use of maximum number of planes for routing a permutation request. If there is a connection from $C_{b c}-K$ for which no plane is available then the connection request pattern is recorded as a blocked connection pattern. The blocking probability is then estimated by the ratio of number of connection requests in which the $0-0$ request is blocked to the total number of connection requests generated.

\subsubsection{Simulation results}

The simulation results for upper bound on blocking probability of VSOB networks with link failures under different crosstalk constraints are given below. 


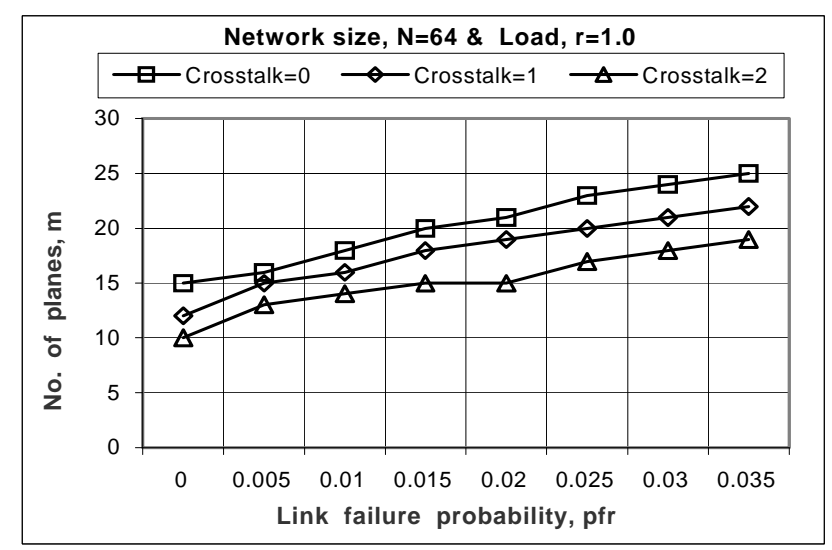

Fig. 4. Maximum number of planes required to make VSOB network nonblocking with a given crosstalk.

Fig. 4 shows that if we allow small amount of crosstalk then the number of planes required to making the VSOB networks nonblocking decreases.

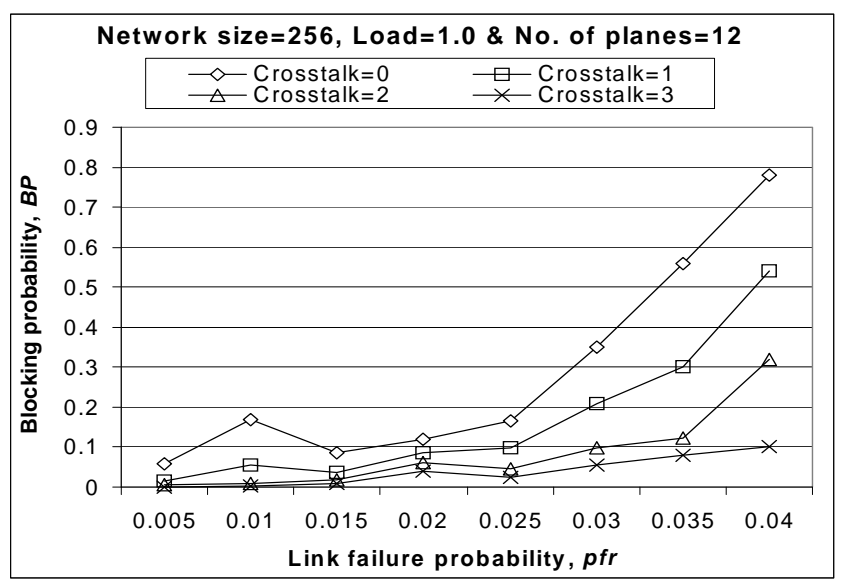

Fig. 5. Blocking probability decreases for certain range of linkfailures and then increases again.

It is also interesting to note from Fig. 5 that the blocking probability not always increases with the increase of link failure; blocking probability also decreases for certain range of link failures and then increases again.

From Fig. 6, for $N=256, p f r=0.01, r=1.0, m=20$; if we allow 3 crosstalk then blocking probability decreases to zero. This picture derive the fact that if we allow small amount of crosstalk then the number of planes required to make the VSOB networks nonblocking decreases dramatically. 


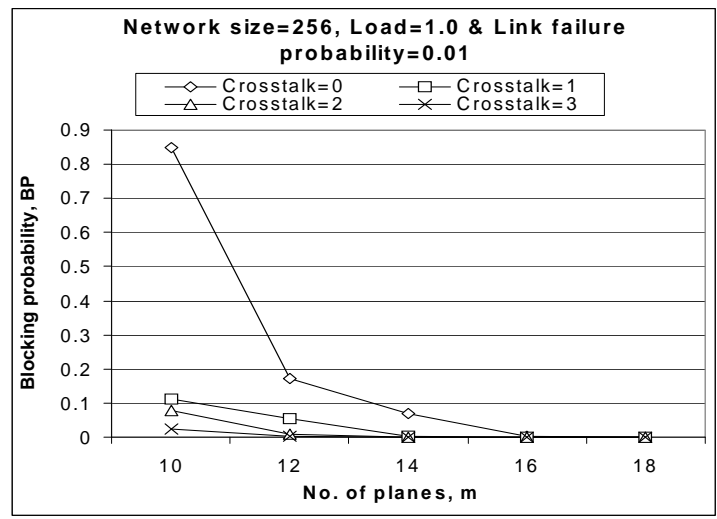

Fig. 6. Blocking probability decreases dramatically with the increase of crosstalk.

Fig. 7 shows that blocking probability decreases dramatically with crosstalk for different load. For $N=128, m=6, p f r=0.02 \& r=0.8$; if we allow 2 crosstalk then blocking probability decreases $67 \%$.

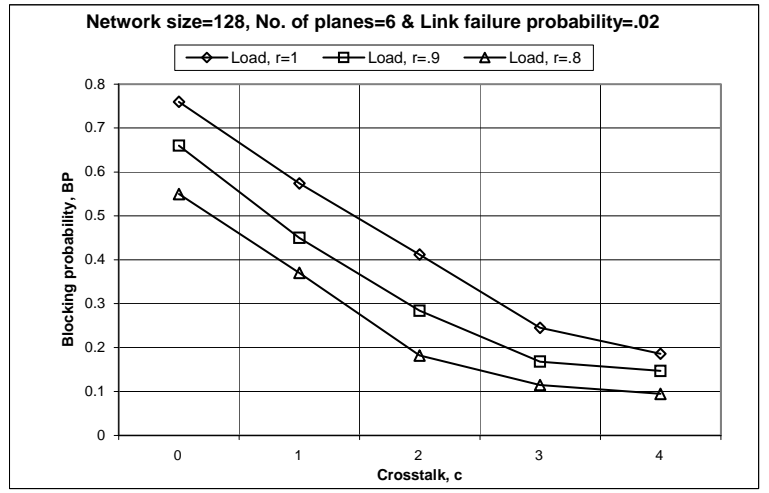

Fig. 7. Crosstalk versus blocking probability for different load.

\subsection{Worst case scenario}

In the above simulation, the traffic generation module randomly generates a permutation request. In that type permutation, the probability of worst-case permutation generation is very small. Jiang et al. in [7] have noticed that there are some permutations which results in maximum blocking connections with the tagged path. For example, permutation $\left(\begin{array}{llllllll}0 & 1 & 2 & 3 & 4 & 5 & 6 & 7 \\ 0 & 4 & 2 & 3 & 1 & 5 & 6 & 7\end{array}\right)$ has 4 such connections that result in blocking with tagged path. Therefore, we need maximum 5 banyan planes to make the network nonblocking. This is 
the worst-case scenario. However, the number of such permutations is not so high. In paper [7], the probability of worst-case scenario is given by

$$
\begin{gathered}
P_{\text {worst }}=2.57 \times \exp ^{-10} \text { for } N=64, r=0.9, \\
P_{\text {worst }}=2.45 \times \exp ^{-20} \text { for } N=128, r=0.9 .
\end{gathered}
$$

So we only generate a subset of all possible worst-case permutations and the simulation results for that case is given below:

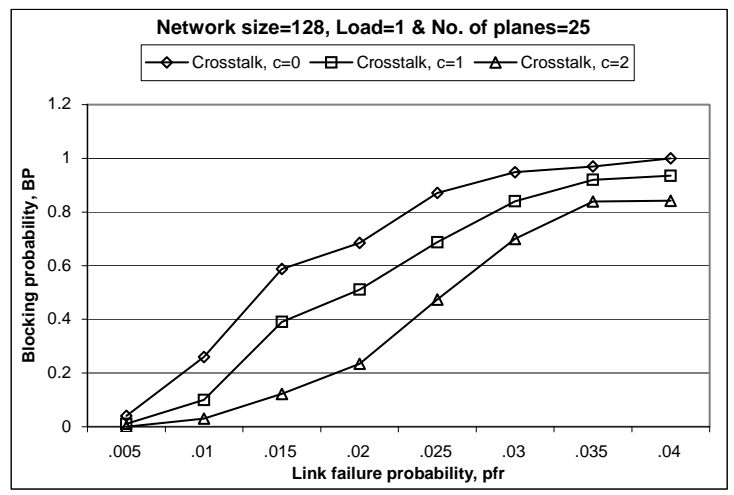

Fig. 8. Link failure probability versus blocking probability for different crosstalk.

Fig. 8 shows that blocking probability gradually increases with link failure probability for different crosstalk. For $p f r=0.03-0.02$; BP decreases $28 \%$ for $c=0$, BP decreases $66 \%$ for $c=2$.

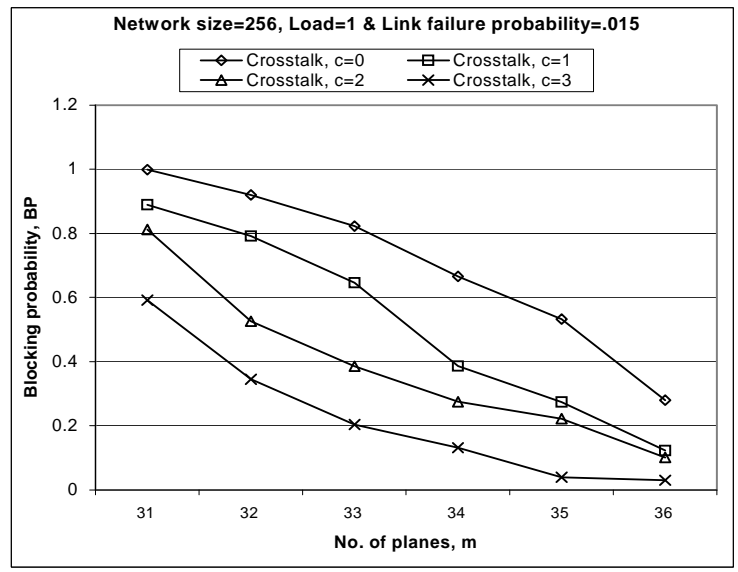

Fig. 9. Number of planes versus blocking probability for different crosstalk. 
Fig. 9 shows that blocking probability decreases dramatically with plane. For $m=31$ 33, BP decreases $17 \%$ for $c=0$, BP decreases $52 \%$ for $c=2$.

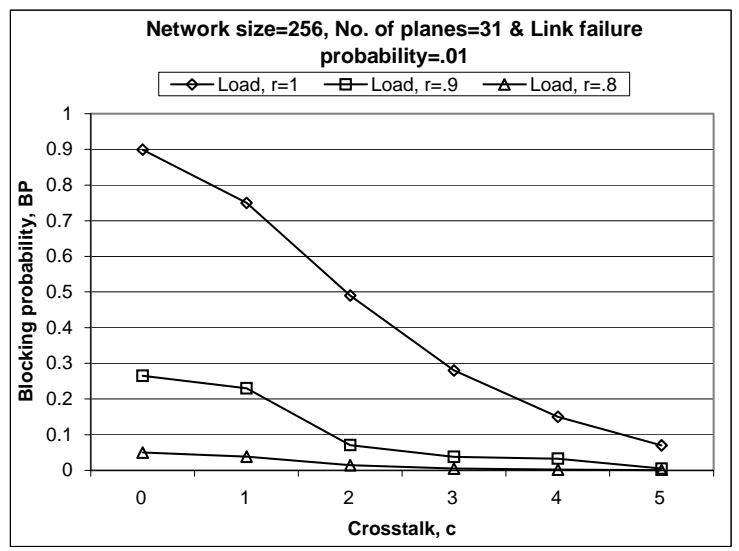

Fig. 10. Crosstalk vs blocking probability for different load under worst-case permutation.

The effect on blocking probability with various crosstalk for a fixed network size, link failure probability and number of planes is shown in Fig. 10. The effect on blocking probability depends on load; if we allow 2 crosstalk then BP decreases $45 \%$ for $r=1$, BP decreases $72 \%$ for $r=0.8$.

\section{Conclusion}

We have presented the results of upper bound on number of planes required to make the VSOB networks nonblocking having link-failures and given certain degree of crosstalk constraint. The simulated results in section 3 can provide network developers with a guidance of quantitatively determining the effects of different degree of crosstalk and number of planes on the overall blocking behaviors of VSOB networks having linkfailures. The results can also show how both the crosstalk and link failures add a new dimension to the performance analysis of VSOB networks, and the effect of crosstalk on hardware cost.

\section{References}

1. H. S. Hinton, An introduction to photonic switching fabrics (Plenum, New York, 1993).

2. V. R. Chinni, T. C. Huang, P. -K. A. Wai, C. R. Menyuk, and G. J. Simonis, IEEE/OSA Journal of Lightwave Technology 13 (7), 1530 (1995). doi:10.1109/50.400714

3. G. R. Goke and G. J. Lipovski, Proc. of 1st Annual Symposium Computer Architecture (1973) pp. 21-28.

4. F. T. Leighton, Introduction to parallel algorithms and architectures: Arrays, Trees, Hypercubes (Morgan Kaufmann, New York, 1992).

5. J. H. Patel, Performance of processor-memory interconnections for multiprocessors, IEEE Transactions on Computers C-30, 771 (1981). doi:10.1109/TC.1981.1675695 
6. M. M. Vaez and C. -T. Lea, IEEE Trans. Commun. 48 (2), 316 (2000). doi:10.1109/26.823564

7. X. Jiang, H. Shen, Md. Mamun-ur-R. Khandker, and S. Horiguchi, Blocking Behavior of Crosstalk free Optical Banyan Networks on Vertical Stacking, IEEE/ACM Transactions on Networking 11 (6), 2003.

8. Rajib Ramaswami and Kumer N. Sivarajan, Optical networks: A Practical Perspective, (Morgan Kaufmann Publishers, San Francisco, USA, ISBN:1-55860-655-6).

9. C. $\mathrm{Yu}, \mathrm{X}$. Jiang and S. Horiguchi, International Journal of Computational Science and Engineering (IJCSE) 2 (1-2), 81 (2006).

10. Xiaohong Jiang, H. Shan, and S. Horiguchi, Bloaking Probability of Vertically Stacked Optical Banyan Networks Under Random Routing, Proc. of IEEE GLOBECOM 2003 (IEEE CS Press, 2004) pp. 2493-2497.

11. Xiaohong Jiang, H. Shan, S. Horiguchi, and P. H. Ho, IEEE Journal of Selected Areas on Communications 23 (8), 1620 (2005). doi:10.1109/JSAC.2005.851754 